\title{
Design of Optically Transparent Antenna with Directional Radiation Patterns
}

\author{
Yuan Yao, ${ }^{1,2}$ Wanzhong Chen, ${ }^{1}$ Xiaodong Chen, ${ }^{2}$ and Junsheng Yu ${ }^{1}$ \\ ${ }^{1}$ School of Electronic Engineering, Beijing University of Posts and Telecommunications, Beijing, China \\ ${ }^{2}$ School of Electronic Engineering and Computer Science, Queen Mary University of London, London, UK
}

Correspondence should be addressed to Yuan Yao; yaoy@bupt.edu.cn

Received 2 April 2017; Revised 29 June 2017; Accepted 6 July 2017; Published 3 August 2017

Academic Editor: Larbi Talbi

Copyright (C) 2017 Yuan Yao et al. This is an open access article distributed under the Creative Commons Attribution License, which permits unrestricted use, distribution, and reproduction in any medium, provided the original work is properly cited.

An optically transparent antenna with directional radiation pattern is proposed in this paper. This antenna consists of a radiation patch above the transparent glass substrate and a frequency selective surface acting as the ground of microstrip antenna on the back of the glass. The frequency selective surface (FSS) performs well as ground in the working frequency range to achieve directional radiation pattern. A SMA connector is used to feed the antenna. The antenna can operate at the on-board dedicated short-range communication band from $5.725 \mathrm{GHz}$ to $5.85 \mathrm{GHz}$. Simulations and measurements are carried out to study the antenna performances in terms of impedance matching, gain, and radiation patterns. Both of the simulation and measurement results are shown to illustrate the good performance of the proposed antenna. It is very suitable for car network communication scene.

\section{Introduction}

At the end of the last century, NASA scientists pioneered the idea of using an optically transparent film to create an antenna [1]. In 2000, the Outaleb research team at Rennes First University in France first proposed the idea of using the ITO thin films to produce optically transparent antennas [2]. So far, optically transparent antennas have been studied for more than a decade. As we know, an optically transparent antenna can be integrated on the surface of other items with almost completely transparent characteristic and will not affect the appearance of goods, which is the unique advantage of optically transparent antennas [3].

At present, there are four kinds of materials that can be used to make optically transparent antennas: the first kind is a transparent metal oxide film, for example, indium tin oxide film (ITO film). In the antenna design, a compromise must be made between the light transmittance and surface impedance [4-6]. The second is the AgHT series multilayer thin film. Compared with the ITO film, the AgHT film has better conductivity and worse transmittance [7]. The third is ultrathin metal film made of copper, silver, gold, and other metal conductors. Antennas made of this material have high radiation efficiency. But the light transmittance is pretty low [8].
The fourth is metal mesh antenna, which has higher radiation efficiency, whose light transmittance is higher than that of the ultrathin metal film. But the requirements for the processing technology of this material are very high [911]. Considering the light transmittance, conductivity, and processing technology of the four materials, ITO film is the best choice. ITO film is also the most widely used material in the research and design of optically transparent antennas.

During a ten-year development, the study of materials that can be used to make transparent antennas has been very comprehensive and extensive. The basic principles of optically transparent antennas had been mastered. Nowadays, the applications of optically transparent antennas in actual scenes attract more attention. There are already some papers that proposed optically transparent antenna structures for a variety of practical scenarios [12-15]. An optically transparent GPS antenna integrated in a car's windshield was proposed by Laser Antenna in Australia [16]. The optically transparent RFID reader antenna for smart fitting room application was proposed by the same authors of this paper [17]. But all of the optically transparent antennas cannot achieve directional radiation [18], which makes these antennas have low effective utilization of radiated power, and hence confidentiality is not good. 


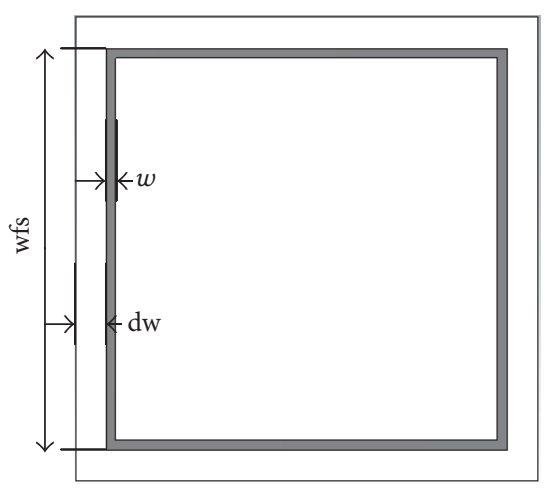

FIgURE 1: The structure of the unit of the square ring FSS.

This paper proposed a structure to realize the directional radiation of optically transparent antennas and designed an optically transparent antenna with directional radiation patterns for car network application scenarios. The proposed antenna is a microstrip antenna with the radiation patch made of transparent ITO film, the substrate made of transparent glass, and the ground of the antenna made of a square ring band resistance frequency selection surface (FSS). The FSS ground has good light transmittance and can reflect the electromagnetic waves radiated from the antenna totally and achieve directional radiation. The antenna works at the on-board dedicated short-range communication band $(5.725-5.850 \mathrm{GHz})$. Simulation and measurement results are shown to illustrate the good performance of the antenna. The results showed that the performance of the proposed antenna is pretty good and it is suitable for car network application scenarios.

\section{FSS Design}

In this proposed antenna design, the square ring FSS structure is used to replace the metal ground of the microstrip antenna, so that the ground of the antenna can reflect the electromagnetic waves in the antenna operating frequency band and has good light transmittance at the same time.

Figure 1 shows the structure of the FSS unit. The main structure parameters of the square ring FSS are the length of the square (wfs), the width of the square ring $(w)$, and the distance between the FSS units $(2 * \mathrm{dw})$. These three parameters together determine the performance of FSS. The main factor determining its total reflection band is the length of the square (wfs); when the circumference of the square ring FSS unit is close to one wavelength of the received electromagnetic wave, the frequency selective surface will reflect the electromagnetic wave back.

The FSS is simulated and optimized by CST electromagnetic simulation software. The square ring unit is simulated under the periodic boundary condition, which means the simulated FSS has an infinite number of FSS units. The FSS is excited by an incident wave which is one of the excitation ports in CST software. S11 of the square ring band stop FSS under the periodic boundary condition is shown in Figure 2. When wfs $=8.4 \mathrm{~mm}, w=0.2 \mathrm{~mm}$, and $\mathrm{dw}=2 \mathrm{~mm}$, the FSS

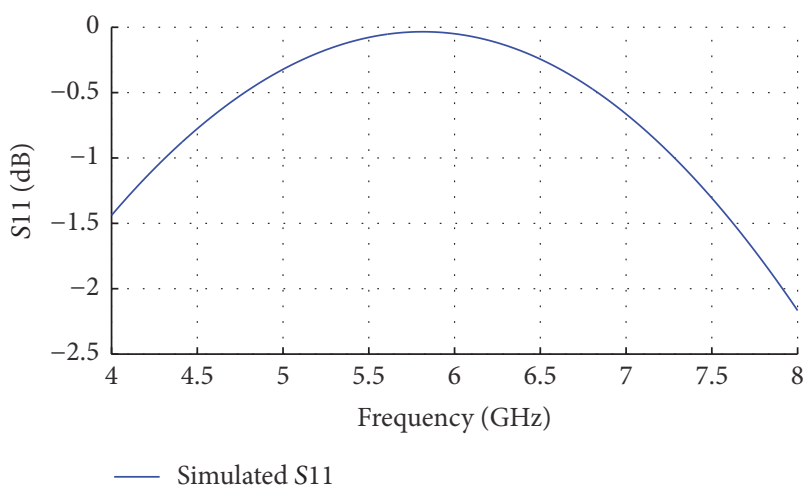

FIGURE 2: S11 of the square ring FSS under the periodic boundary condition.

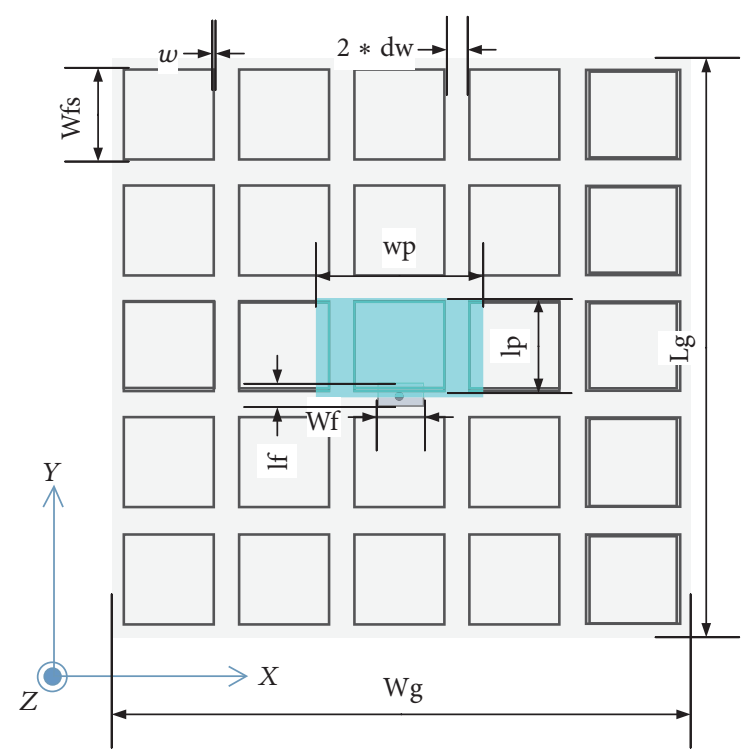

FIgURE 3: The overall view of the whole antenna.

has good total reflection characteristic in the short-range communication band. Since the width of the square ring is very small, the metal part in the FSS structure is relatively less, which means the FSS has good light transmittance.

\section{Antenna Design}

The structure of the proposed antenna is shown in Figures 3 and 4 . Figure 3 shows the overall view of the whole antenna and Figure 4 shows the side view of the proposed antenna. The top layer is the radiation patch made of ITO film, whose surface impedance is $7 \Omega / s q$. The length of the patch is lp and the width of the patch is wp. The middle layer is the transparent glass substrate, whose permittivity is 4.6 , loss tangent is 0.0036 , and thickness is $1 \mathrm{~mm}$. The length of the glass substrate is $\mathrm{Lg}$ and the width of the glass substrate is $\mathrm{Wg}$. The bottom layer is the $5 \times 5$ square ring FSS structure, which is metal sputtered onto the glass substrate. The feed point is put at the midpoint of the lower side of the patch. A metal sheet was put on the FSS to achieve better feeding, 


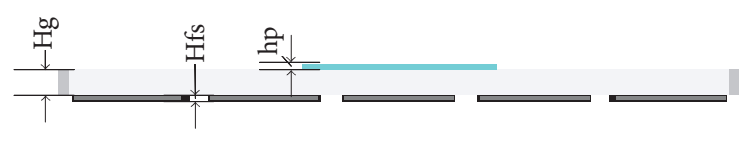

FIGURE 4: The side view of the proposed antenna.

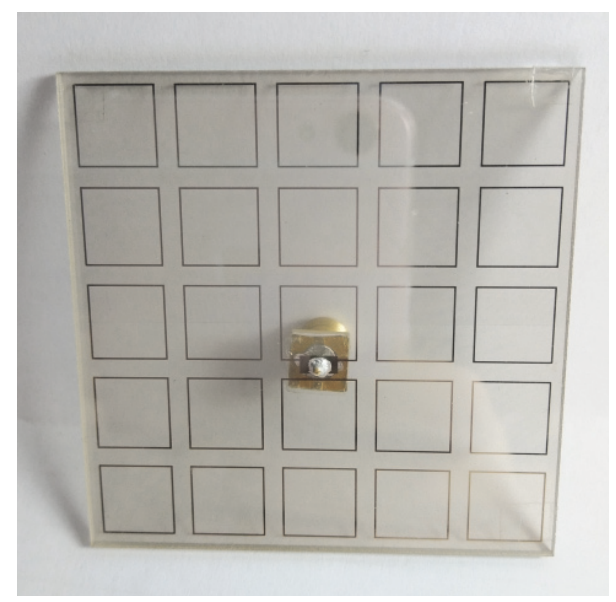

FIGURE 5: Overall view of the fabricated proposed antenna.

TABLE 1: Parameters of the proposed antenna (units: $\mathrm{mm}$ ).

\begin{tabular}{lc}
\hline Wg & 52 \\
Lg & 52 \\
Hg & 1 \\
wp & 12 \\
lp & 8.85 \\
Hp & 0.00025 \\
wfs & 8.4 \\
Hfs & 0.003 \\
$w$ & 0.2 \\
dw & 2 \\
wf & 4 \\
lf & 2 \\
\hline
\end{tabular}

whose length is lf and width is wf. The designed antenna is expected to work at the on-board dedicated short-range communication band from $5.725 \mathrm{GHz}$ to $5.85 \mathrm{GHz}$.

Figures 5 and 6 show the fabricated proposed antenna. The antenna is fed by a SMA connecter with $50 \Omega$ impedance. The finally chosen dimensions of the proposed antenna are illustrated in Table 1. It is obvious that the antenna has magnificent light transmittance. We have measured the light transmittance of the antenna, which is about $80 \%$ in the area of the patch and $84 \%$ around the patch.

\section{Parameter Study of the Antenna}

Parametric studies were carried out to characterize the proposed antenna, where, unless otherwise stated, only a single parameter was changed, while the others were kept constant as in Table 1.

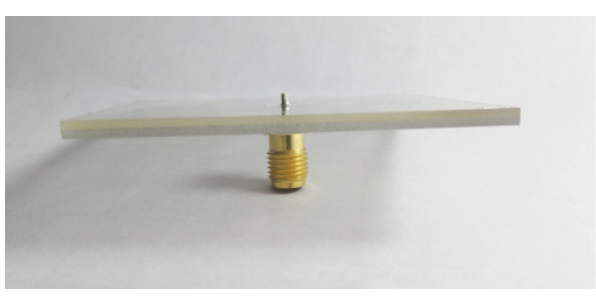

FIGURE 6: Side view of the fabricated proposed antenna.

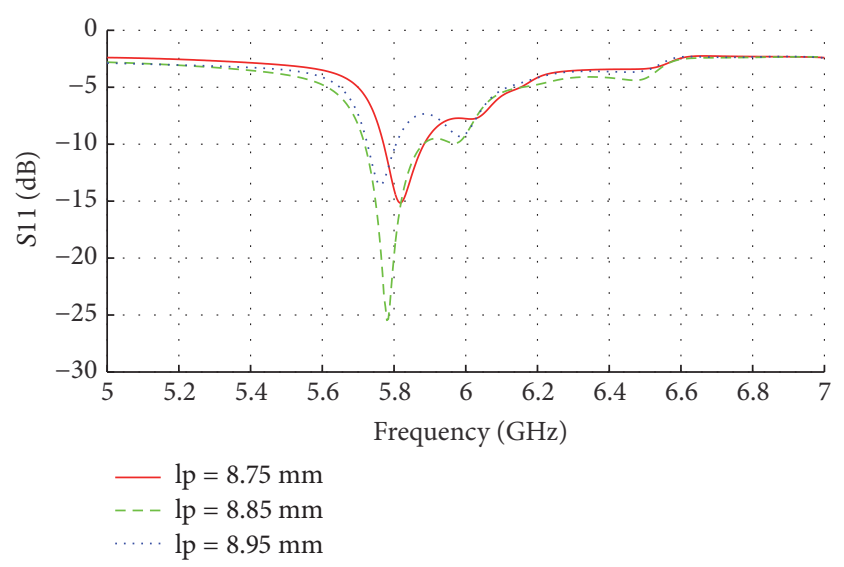

FIgURE 7: The optimization of length of the radiation patch.

4.1. Radiation Patch Design (Main Parameters: Ip and $w p$ ). In order to verify the influence of the parameters of the designed antenna on the overall characteristics of the antenna, we studied the parameters of the antenna in CST electromagnetic simulation software.

In this antenna design, the length and width of the radiation patch determine the resonant point and impedance bandwidth. Figure 7 shows that the resonant point of the antenna decreases as the length of the radiating patch (lp) increases. When $\mathrm{lp}=8.85 \mathrm{~mm}$, the antenna resonates at $5.8 \mathrm{GHz}$, and the working frequency band whose $S 11$ is below $-10 \mathrm{~dB}$ is $5.725-5.85 \mathrm{GHz}$. Figure 8 shows that, with the width of the patch (wp) increasing, the antenna resonant frequency is declining. When $w p=12 \mathrm{~mm}$, the antenna resonates at $5.8 \mathrm{GHz}$ and can completely cover the on-board short-range communication frequency band.

4.2. Square Ring FSS Design (Main Parameters: $w f s, w$, and $d w)$. In this design, the square ring frequency selective surface is used to replace the ground of the microstrip antenna, which can reflect the electromagnetic wave back in the antenna operating frequency band. Figure 9 shows that as the length of the square (wfs) increases, the resonant frequency band of the antenna decreases. When the circumference of the square ring FSS unit is about the resonant wavelength of the microstrip antenna on board, that is, when wfs = $8.4 \mathrm{~mm}$, the antenna just resonates at $5.8 \mathrm{GHz}$. $S 11$ are less than $-10 \mathrm{~dB}$ in the on-board short-range communication band $(5.725-5.85 \mathrm{GHz})$.

Figure 10 shows that, with the increase of the width of the square ring $(w)$, the resonant frequency is increasing. When 


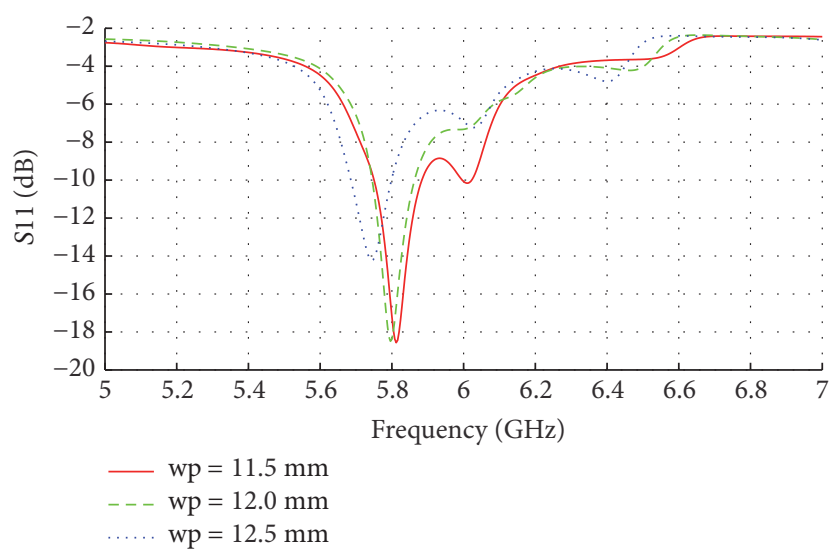

FIGURE 8: The optimization of width of the radiation patch.

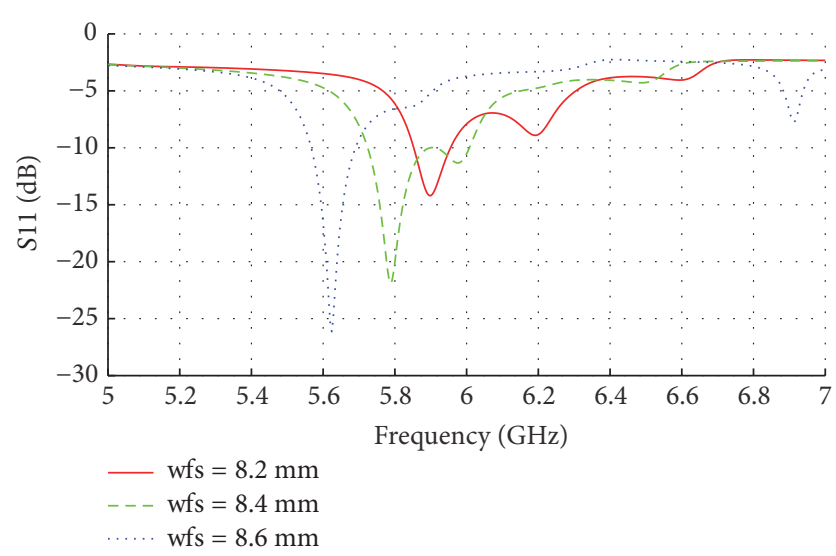

FIGURE 9: The optimization of length of the square ring unit.

$w=0.2 \mathrm{~mm}$, the antenna just resonates at $5.8 \mathrm{GHz}$, and the frequency band is in the range $5.725-5.85 \mathrm{GHz}$ and $S 11$ are less than $-10 \mathrm{~dB}$, which meet the requirements of car network communication.

Figure 11 shows that the distance between the FSS units $(2 * \mathrm{dw})$ increases, the antenna's impedance bandwidth becomes narrow, and the resonant frequency of the antenna increases. When $\mathrm{dw}=2.0 \mathrm{~mm}$, the antenna resonates at $5.8 \mathrm{GHz}$ and $S 11$ are less than $-10 \mathrm{~dB}$ over the on-board shortrange communication band $(5.725-5.85 \mathrm{GHz})$.

\section{Simulation and Measurement Results}

The measurements were carried out in a chamber.

5.1. S-Parameter. Figure 12 shows the simulated and measured $S 11$ of the proposed antenna. The solid line is the simulation result, and the dashed line is the measurement result. It can be seen that the measured result agrees well with the simulation result, and the trends of the two are the same. In the on-board short-range communication band $(5.725-5.85 \mathrm{GHz}), S 11$ are less than $-10 \mathrm{~dB}$, which means the antenna has good impedance matching characteristic and can meet the requirement of car network communication.

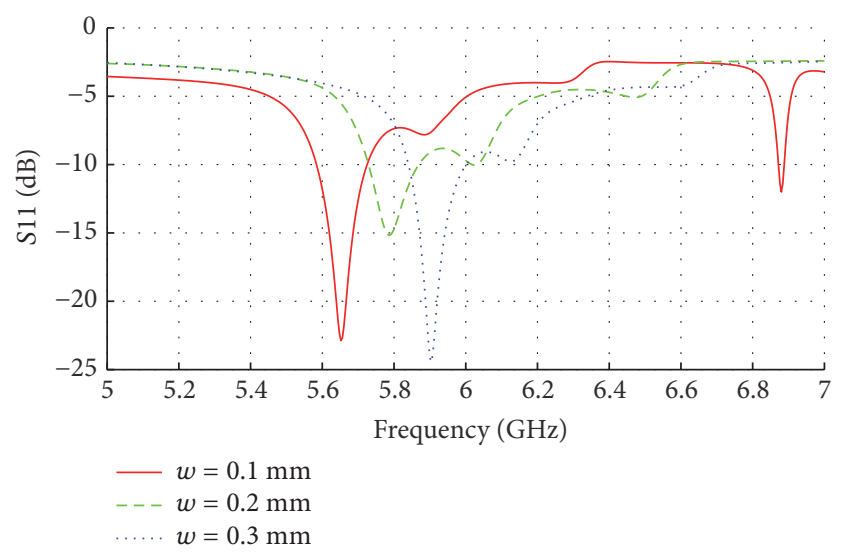

FIGURE 10: The optimization of width of the square ring.

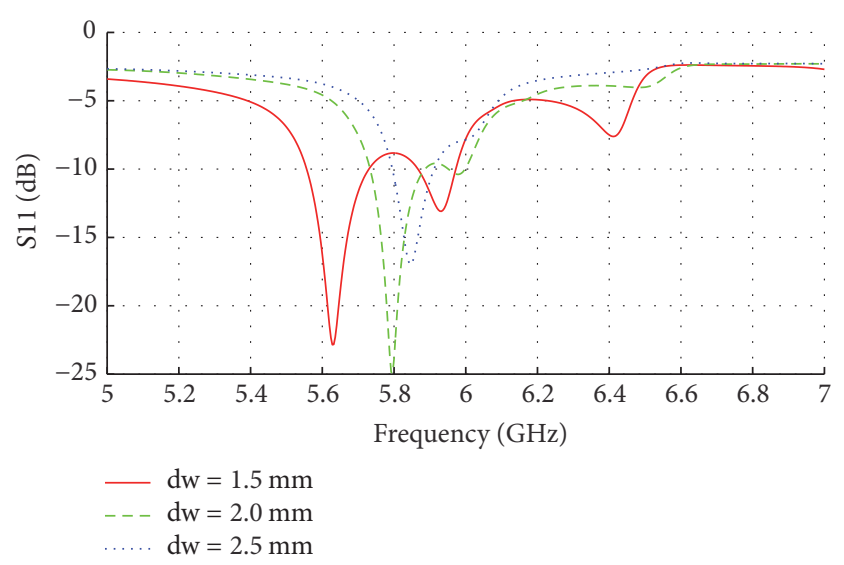

FIGURE 11: The optimization of distance between the units.

5.2. Gain. Figure 13 shows the gain of the designed antenna. The solid line is the actual measurement result, and the dotted line is the simulation result. The overall trends of the two lines are basically the same; the difference between them is due to fabrication error and ITO model accuracy in simulation software. It can be seen that, in the on-board short-range communication band $(5.725-5.85 \mathrm{GHz})$, the radiation gain of the antenna is around $-6 \mathrm{dBi}$. This is because the patch of the antenna is made of indium tin oxide film, and it has a high surface impedance, which means the conductivity is not very good. Thus, the radiation gain of the antenna produced by this material is low. However, the $-6 \mathrm{dBi}$ gain is also enough for our specific application, which is on-board short-range communication.

5.3. Radiation Patterns. Figures $14,15,16,17,18$, and 19 show the radiation patterns of the designed antenna in $X O Z$ plane and $Y O Z$ plane in the on-board short-range communication band $(5.725-5.85 \mathrm{GHz})$. The solid line is the measurement result and the dashed line is the simulation result. It can be seen that, in the working frequency band $5.725-5.85 \mathrm{GHz}$, the antenna has good directional radiation performance. The maximum radiation direction is $+Z$ direction, and it is almost perpendicular to the plane where the antenna is located. 


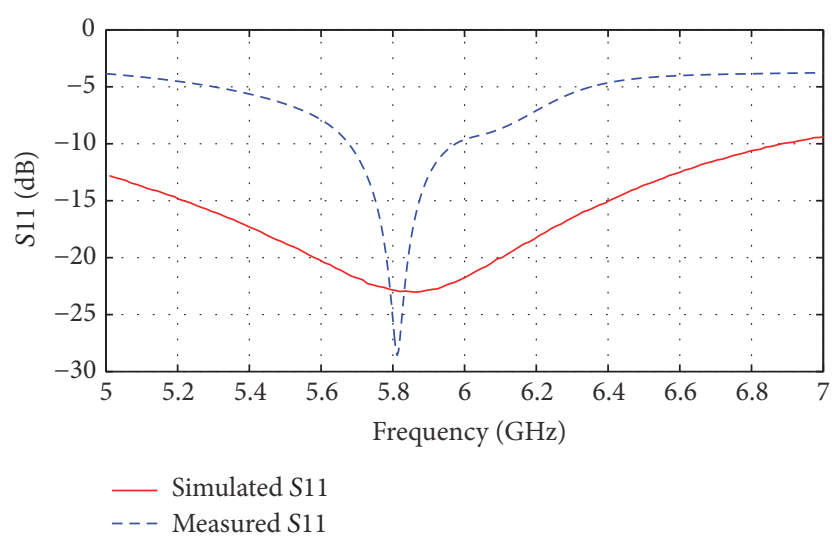

FIGURE 12: Simulated and measured $S 11$ of the proposed antenna.

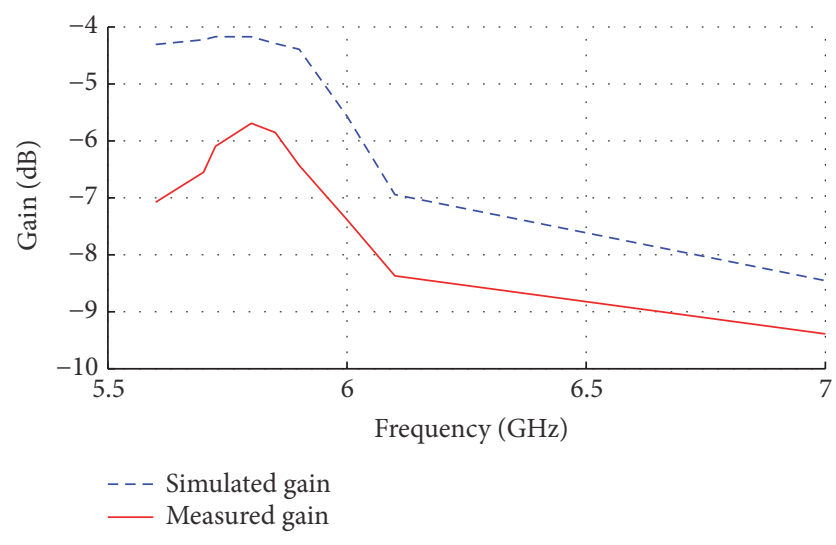

FIGURE 13: Simulated and measured gain of the proposed antenna.

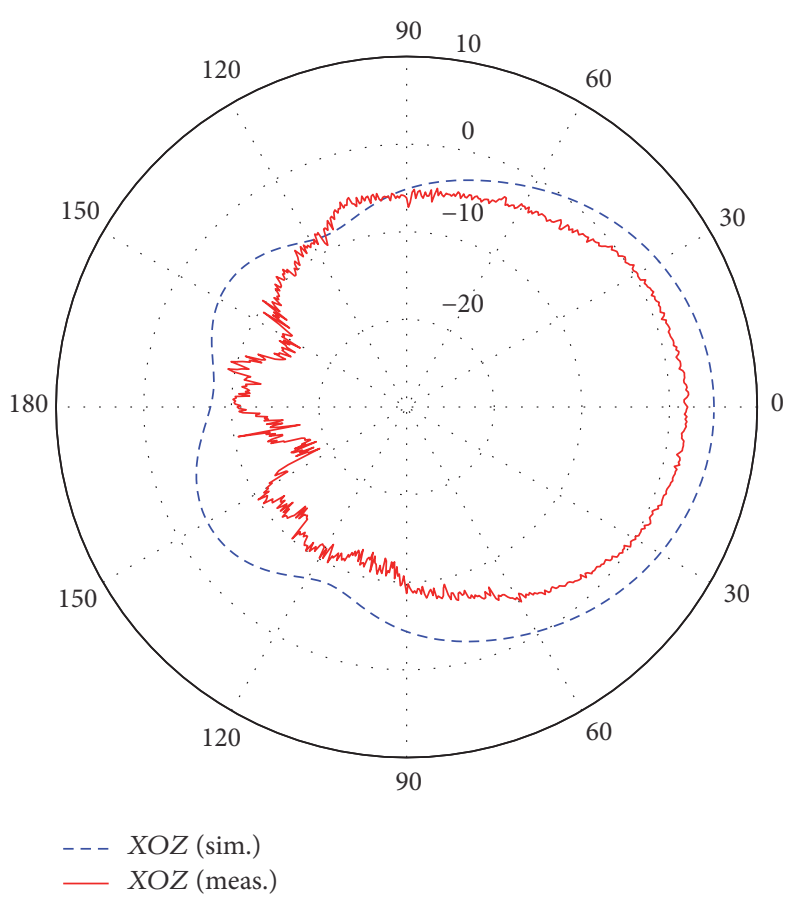

FIGURE 14: Radiation pattern of the antenna in $X O Z$ plane at $5.725 \mathrm{GHz}$.

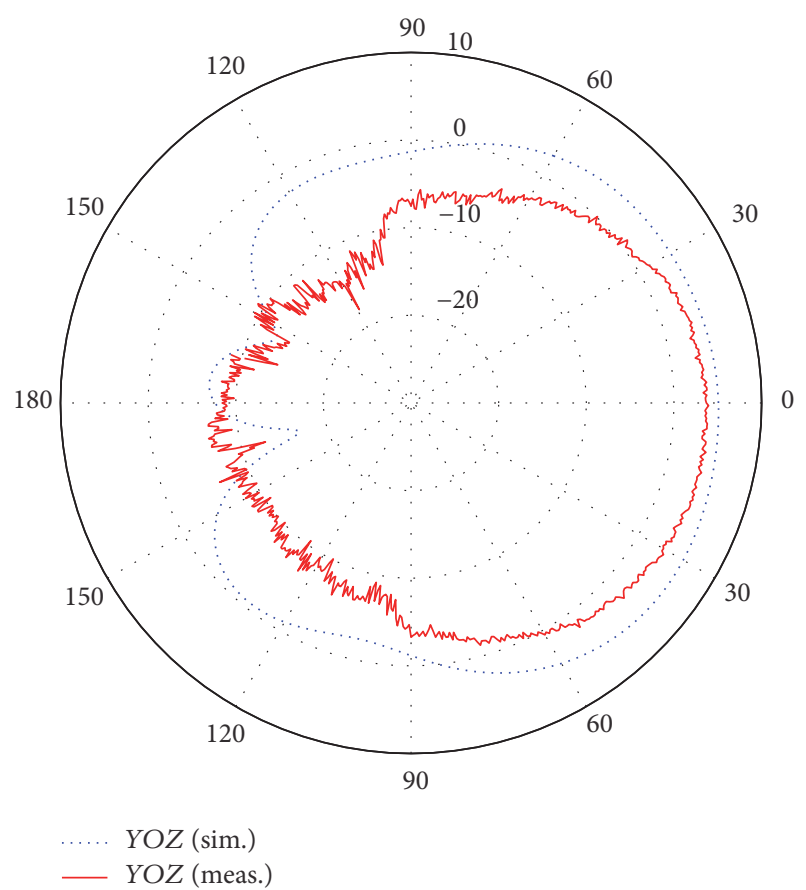

FIGURE 15: Radiation pattern of the antenna in YOZ plane at $5.725 \mathrm{GHz}$.

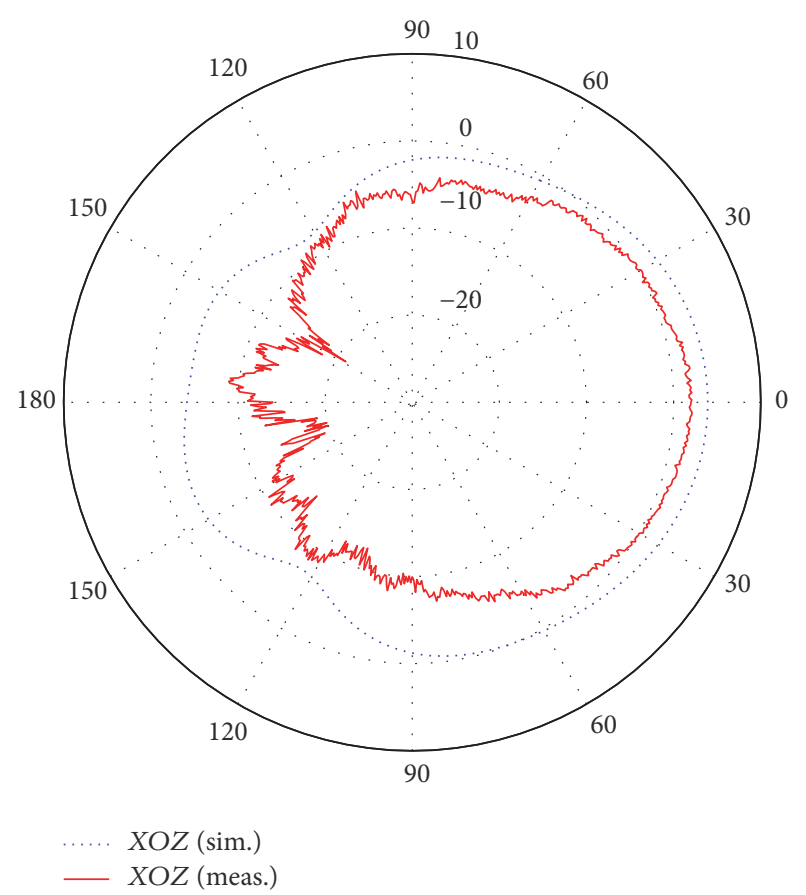

FIgURE 16: Radiation pattern of the antenna in $X O Z$ plane at $5.8 \mathrm{GHz}$.

Therefore, when the antenna is stuck to the windows of a car in the car networking scene, it can keep the radiated energy outside of the vehicle effectively and avoid radiation damage to people in the vehicle as much as possible. It can be seen that there are some differences between the simulation results and the measurement results. There are two main reasons for 


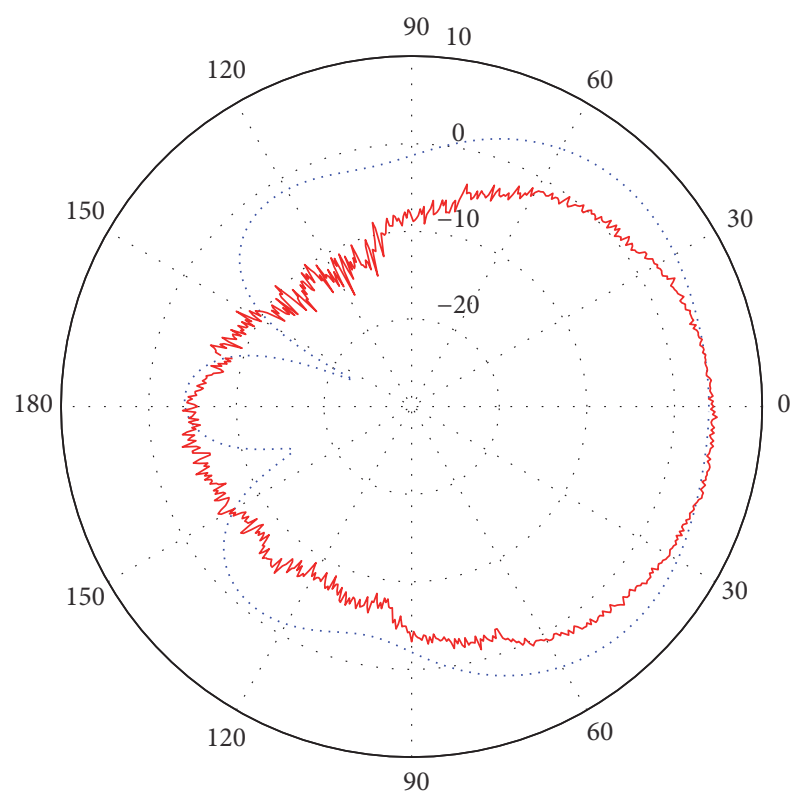

YOZ (sim.)

— YOZ (meas.)

Figure 17: Radiation pattern of the antenna in YOZ plane at $5.8 \mathrm{GHz}$

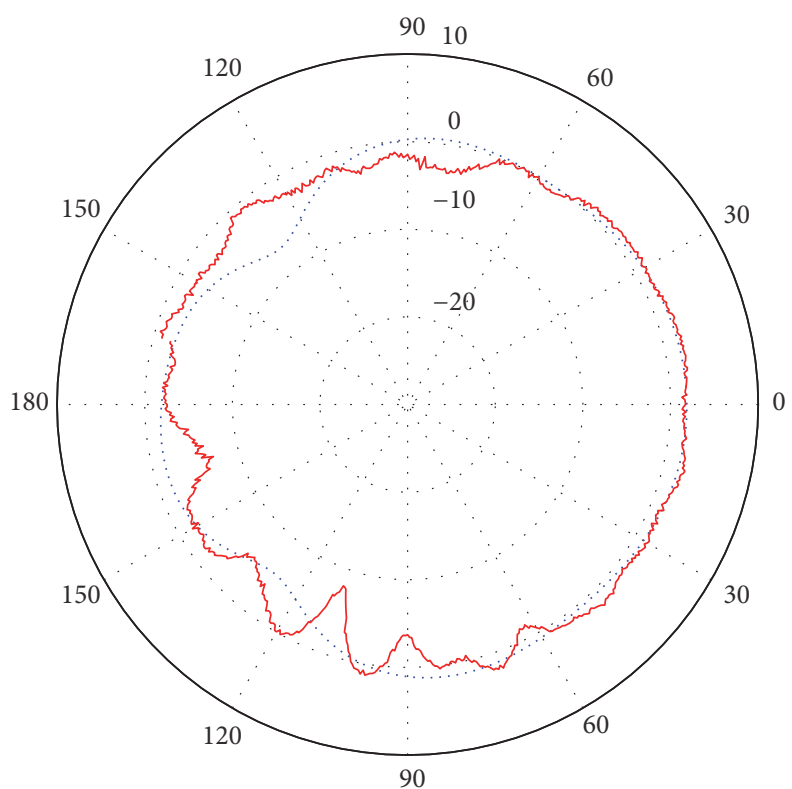

… XOZ (sim.)

— XOZ (meas.)

FIGURE 18: Radiation pattern of the antenna in $X O Z$ plane at $5.85 \mathrm{GHz}$.

these differences. First, the ITO model in CST is not very accurate, and thus there are some differences between the simulation and the actual prototype. Second, the fabrication error can also bring about differences.

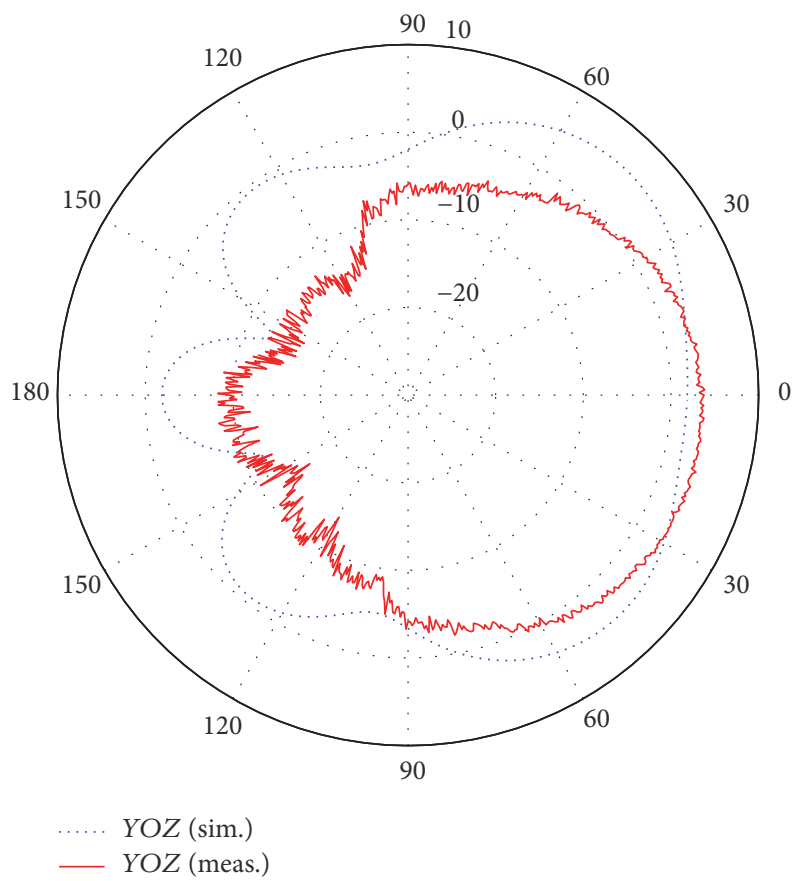

FIGURE 19: Radiation pattern of the antenna in YOZ plane at $5.85 \mathrm{GHz}$.

\section{Conclusion}

In this paper, an optically transparent antenna with directional radiation pattern is designed. The patch of the antenna is made of indium tin oxide film whose surface impedance is $7 \Omega /$ sq. The substrate is transparent glass with a dielectric constant of 4.6 and thickness of $1 \mathrm{~mm}$. In this antenna structure, a $5 * 5$ square ring FSS is selected to replace the ground of a microstrip antenna, so as to realize the optical transparency and directional radiation characteristics. The simulation and measurement results show that the antenna structure has good S11, gain, and radiation performances in the onboard short-range communication band $(5.725-5.85 \mathrm{GHz})$. The directional radiation direction is perpendicular to the antenna. When the antenna is applied to the windows of a car, it is very suitable for the complex and changeable electromagnetic environment. Thus, the proposed antenna is very suitable for car networking scenes.

\section{Conflicts of Interest}

The authors declare that there are no conflicts of interest regarding the publication of this paper.

\section{Acknowledgments}

This work is funded by the China Scholarship Council and the K. C. Wong Foundation.

\section{References}

[1] R. N. Simons and R. Q. Lee, "Feasibility study of optically transparent microstrip patch antenna," in Proceedings of the 
1997 IEEE Antennas and Propagation Society International Symposium. Part 4 (of 4), pp. 2100-2103, July 1997.

[2] N. Outaleb, J. Pinel, M. Drissi, and O. Bonnaud, "Microwave planar antenna with RF-sputtered indium tin oxide films," Microwave and Optical Technology Letters, vol. 24, no. 1, pp. 3-7, 2000.

[3] Q. H. Dao, T. J. Cherogony, and B. Geck, "Optically transparent and circularly polarized patch antenna for K-band applications," in Proceedings of the German Microwave Conference, GeMiC 2016, pp. 247-250, March 2016.

[4] Y. Yao, J. Yu, and X. Chen, "Study on the optically transparent near-field and far-field RFID reader antenna," International Journal of Antennas and Propagation, vol. 2014, Article ID 149051, 5 pages, 2014.

[5] Y. Wang, C. Zhang, and G. Ding, "Fabrication and characterization of ITO thin film resistance temperature detector," in Proceedings of the 7th IEEE International Nanoelectronics Conference, INEC 2016, chn, May 2016.

[6] G. J. Exarhos and X.-D. Zhou, "Discovery-based design of transparent conducting oxide films," Thin Solid Films, vol. 515, no. 18, pp. 7025-7052, 2007.

[7] M. S. A. Rani, S. K. A. Rahim, M. R. Kamarudin, T. Peter, S. W. Cheung, and B. M. Saad, "Electromagnetic behaviors of thin film CPW-Fed CSRR loaded on UWB transparent antenna," IEEE Antennas and Wireless Propagation Letters, vol. 13, pp. 1239-1242, 2014.

[8] C. Mias, "Optically transparent microstrip antennas," in Proceedings of the IEE Colloquium on Antennas for Automotives, pp. 8-8, London, UK.

[9] J. Hautcoeur, F. Colombel, X. Castel, M. Himdi, and E. Motta Cruz, "Optically transparent monopole antenna with high radiation efficiency manufactured with silver grid layer (AgGL)," Electronics Letters, vol. 45, no. 20, pp. 1014-1016, 2009.

[10] J. Hautcoeur, F. Colombel, X. Castel, M. Himdi, and E. MottaCruz, "Radiofrequency performances of transparent ultrawideband antennas," Progress In Electromagnetics Research $C$, vol. 22, pp. 259-271, 2011.

[11] N. Guan, H. Furuya, R. Hosono, H. Tayama, and K. Yamagami, "A see-through wire-grid film antenna for WLAN applications," in Proceedings of the 2012 IEEE Asia-Pacific Conference on Antennas and Propagation, APCAP 2012, pp. 273-274, August 2012.

[12] T. Beltramonte, M. Di Bisceglie, and C. Galdi, "Optimum systems for satellite fire detection," in Proceedings of the 2010 30th IEEE International Geoscience and Remote Sensing Symposium, IGARSS 2010, pp. 506-509, July 2010.

[13] R. M. Taylor, "Space communications," IEEE Spectrum, vol. 29, no. 2, pp. 30-33, 1992.

[14] M. V. T. Heckler, L. A. Greda, and A. Dreher, "Analysis of a navigation antenna installed on a civil airplane," in Proceedings of the 2008 IEEE International Symposium on Antennas and Propagation and USNC/URSI National Radio Science Meeting, APSURSI, July 2008.

[15] S. Voigt, "TWT's in Space - Present status and future applications," in Proceedings of the 2009 IEEE International Vacuum Electronics Conference, IVEC 2009, pp. 53-56, April 2009.

[16] Laser Clear Antennas, http://www.laser-antenna.com/downloads/printable/GPSBrochure777.pdf.

[17] Y. Yao, N. He, W. Chen, J. Yu, and X. Chen, "Novel optically transparent antenna for RFID smart fitting room application," in Proceedings of the Asia-Pacific Microwave Conference, APMC 2015, December 2015.
[18] M. A. Moharram and A. A. Kishk, "Optically transparent reflectarray antenna design integrated with solar cells," IEEE Transactions on Antennas and Propagation, vol. 64, no. 5, pp. 1700-1712, 2016. 


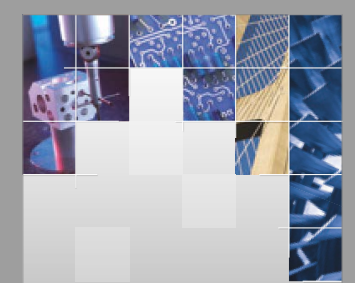

\section{Enfincering}
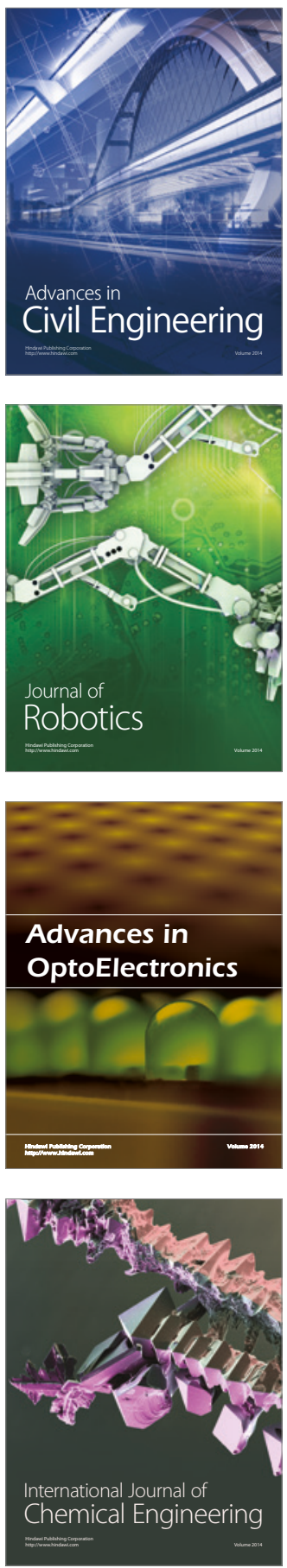

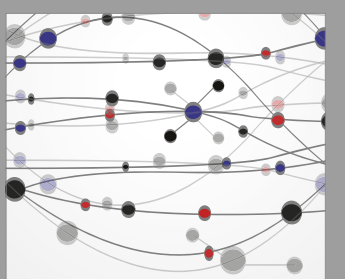

The Scientific World Journal

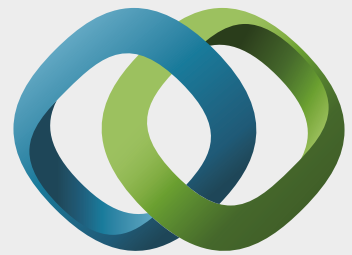

\section{Hindawi}

Submit your manuscripts at

https://www.hindawi.com
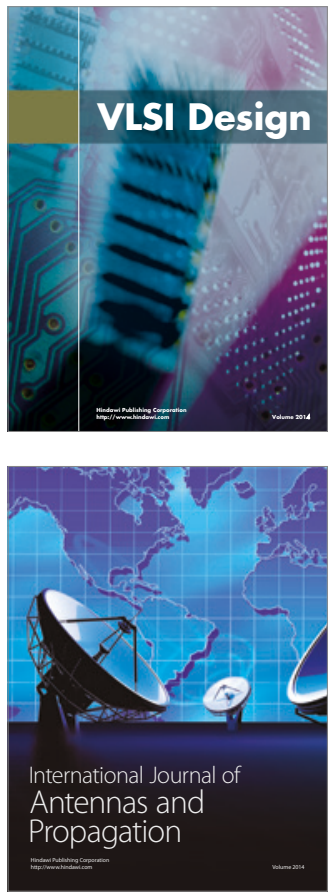

\section{Rotating}

Machinery
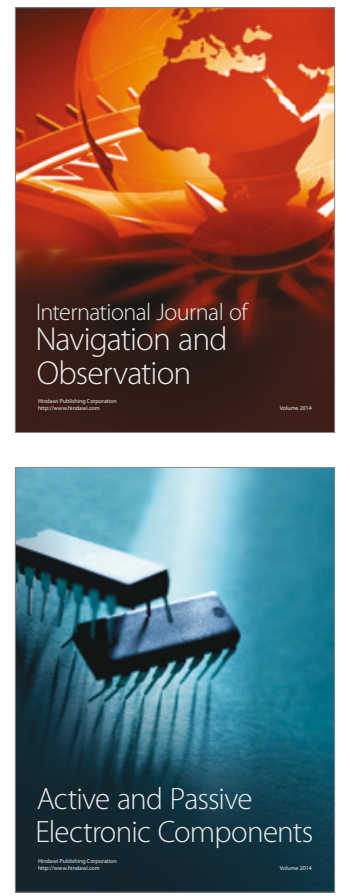
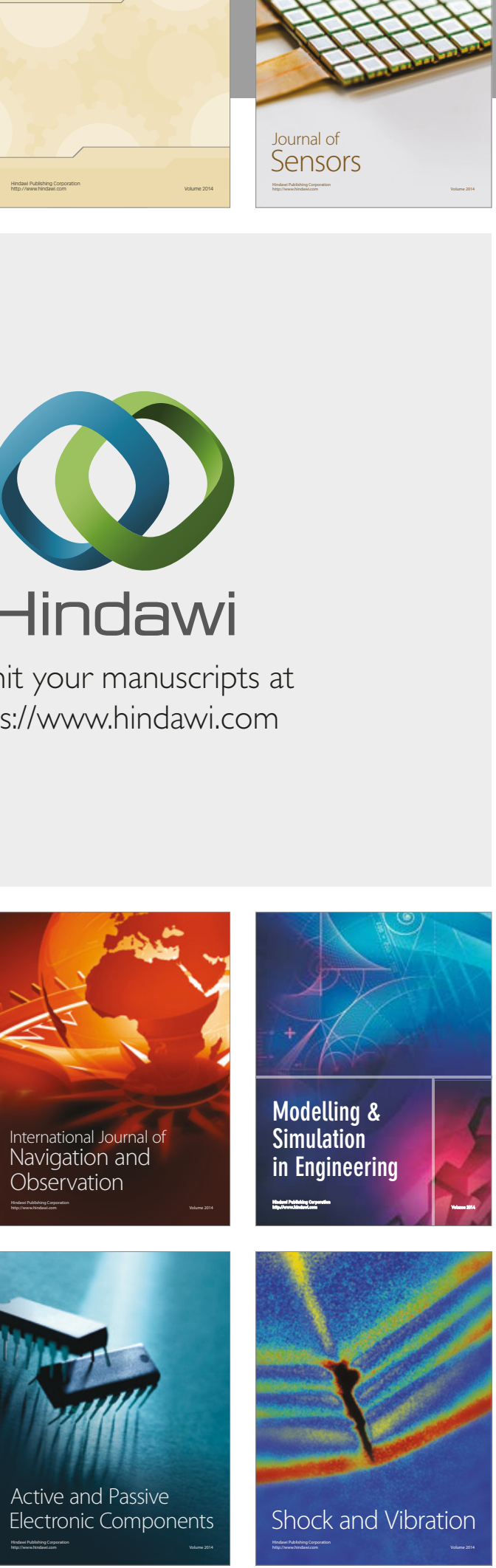
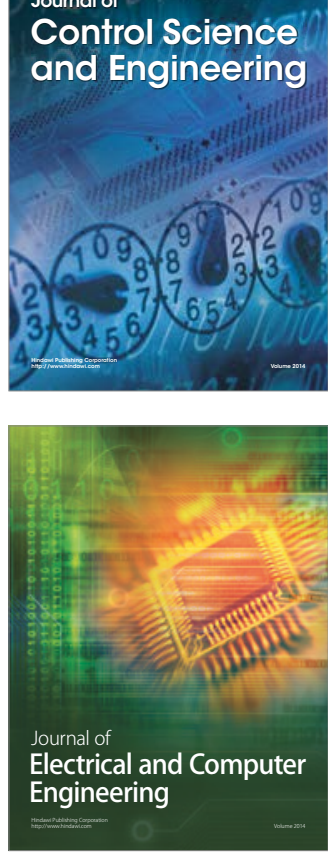

Distributed

Journal of

Control Science

and Engineering
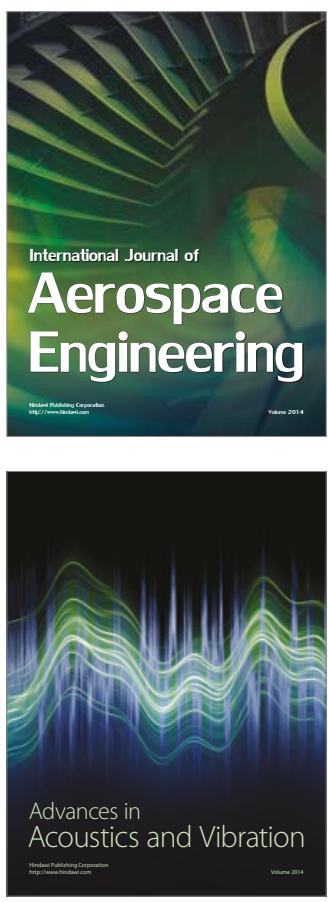

Sensor Networks 